\title{
CHARACTERISATIONS OF GEODESIC HYPERSPHERES IN A NON-FLAT COMPLEX SPACE FORM
}

\author{
SADAHIRO MAEDA \\ Department of Mathematics, Saga University, Saga 840-8502, Japan \\ e-mail:smaeda@ms.saga-u.ac.jp \\ TOSHIAKI ADACHI \\ Department of Mathematics, Nagoya Institute of Technology, Gokiso, Nagoya 466-8555, Japan \\ e-mail: adachi@nitech.ac.jp \\ and YOUNG HO KIM \\ Department of Mathematics, Teachers College, Kyungpook National University, Taegu 702-701, Korea \\ e-mail:yhkim@knu.ac.kr
}

(Received 12 November 2011; accepted 9 May 2012; first published online 2 August 2012)

\begin{abstract}
Totally $\eta$-umbilic real hypersurfaces are the simplest examples of real hypersurfaces in a non-flat complex space form. Geodesic hyperspheres in this ambient space are typical examples of such real hypersurfaces. We characterise every geodesic hypersphere by observing the extrinsic shapes of its geodesics and using the derivative of its contact form.
\end{abstract}

2000 Mathematics Subject Classification. Primary 53B25, Secondary 53C40.

1. Introduction. An $n$-dimensional non-flat complex space form $\widetilde{M}_{n}(c)$ is a complex $n(\geqq 2)$-dimensional complete and simply connected Kähler manifold of nonzero constant holomorphic sectional curvature $c$. It is holomorphically isometric to either an $n$-dimensional complex projective space $\mathbb{C} P^{n}(c)$ of constant holomorphic sectional curvature $c$ or an $n$-dimensional complex hyperbolic space $\mathbb{C} H^{n}(c)$ of constant holomorphic sectional curvature $c$ accordingly as $c$ is positive or negative.

In this paper, we study real hypersurfaces isometrically immersed into $\widetilde{M}_{n}(c)$ from two points of view. One is to observe extrinsic shapes of geodesics on a real hypersurface $M$ in the ambient space $\widetilde{M}_{n}(c)$, and the other is to study the contact form $\eta$ on $M$ in $\widetilde{M}_{n}(c)$. In submanifold theory, it is natural to understand the shape of a Riemannian submanifold by observing the extrinsic shapes of geodesics on the submanifold (for example, see $[3,6,7, \mathbf{1 0}, \mathbf{1 2}, \mathbf{1 6}])$. A surface $M$ in $\mathbb{R}^{3}$ is locally either a plane or a standard sphere if every geodesic on $M$ is mapped to a circle in $\mathbb{R}^{3}$ through the isometric immersion. In $\widetilde{M}_{n}(c)$, unfortunately, there exist no real hypersurfaces all of whose geodesics are mapped to circles in $\widetilde{M}_{n}(c)$, because this ambient space admits no totally umbilic real hypersurfaces. However, there do exist real hypersurfaces some of whose geodesics are mapped to circles in $\widetilde{M}_{n}(c)$. Totally $\eta$-umbilic real hypersurfaces are the simplest examples of real hypersurfaces satisfying this geometric property. From the second point of view concerning contact forms, it is known that there exist no real hypersurfaces whose contact form is closed with respect to the exterior derivative on $M$ (cf. [15]). We are hence interested in making a condition on contact forms weaken. We 
investigate the exterior derivatives of contact forms, extrinsic shapes of geodesics and sectional curvatures on totally $\eta$-umbilic real hypersurfaces, and characterise geodesic hyperspheres among all real hypersurfaces in $\widetilde{M}_{n}(c)$.

2. Characterisations. Let $M^{2 n-1}$ be a real hypersurface in a non-flat complex space form $\widetilde{M}_{n}(c)$ with a unit normal local vector field $\mathcal{N}$. It has an almost contact metric structure $(\phi, \xi, \eta,\langle\rangle$,$) induced from the standard Riemannian metric \langle$,$\rangle and$ the complex structure $J$ of the ambient space $\widetilde{M}_{n}(c)$. This quadruple is formed by induced metric and a $(1,1)$-tensor $\phi$, a vector field $\xi$ and a 1-form $\eta$ given by

$$
\langle\phi X, Y\rangle=\langle J X, Y\rangle, \quad \xi=-J \mathcal{N} \quad \text { and } \quad \eta(X)=\langle\xi, X\rangle=\langle J X, \mathcal{N}\rangle .
$$

These $\xi$ and $\eta$ are usually called the characteristic vector field and the contact form on $M$, respectively.

A real hypersurface $M$ of $\widetilde{M}_{n}(c)(n \geqq 2)$ is called totally $\eta$-umbilic if its shape operator $A$ is of the form $A=\alpha I+\beta \eta \otimes \xi$ for some smooth functions $\alpha$ and $\beta$ on $M$. Note that these two smooth functions $\alpha$ and $\beta$ are automatically constant. We usually say a real hypersurface $M$ to be Hopf if its characteristic vector field $\xi$ is principal at each point of $M$. By the definition, we see that every totally $\eta$-umbilic real hypersurface $M$ with $A=\alpha I+\beta \eta \otimes \xi$ is a Hopf hypersurface having two distinct constant principal curvatures $\alpha$ and $\delta=\alpha+\beta$ (cf. $[14,15])$. A totally $\eta$-umbilic real hypersurface of $\widetilde{M}_{n}(c)(n \geqq 2)$ with shape operator $A=\alpha I+\beta \eta \otimes \xi$ is hence locally congruent to one of the following:

(P) a geodesic hypersphere $G(r)$ of radius $r(0<r<\pi / \sqrt{c})$ in $\mathbb{C} P^{n}(c)$,

$\left(\mathrm{H}_{\mathrm{i}}\right)$ a horosphere $H S$ in $\mathbb{C} H^{n}(c)$,

$\left(\mathrm{H}_{\mathrm{ii}}\right)$ a geodesic hypersphere $G(r)$ of radius $r(0<r<\infty)$ in $\mathbb{C} H^{n}(c)$,

$\left(\mathrm{H}_{\mathrm{iii}}\right)$ a tube $T(r)$ of radius $r(0<r<\infty)$ around a totally geodesic holomorphic hyperplane $\mathbb{C} H^{n-1}(c)$ in $\mathbb{C} H^{n}(c)$.

In these cases, $\alpha, \beta$ are as follows:

$$
\alpha=\left\{\begin{array}{ll}
(\sqrt{c} / 2) \cot (\sqrt{c} r / 2), \\
\sqrt{|c|} / 2, \\
(\sqrt{|c|} / 2) \operatorname{coth}(\sqrt{|c|} r / 2), \\
(\sqrt{|c|} / 2) \tanh (\sqrt{|c|} r / 2),
\end{array} \quad \beta= \begin{cases}-(\sqrt{c} / 2) \tan (\sqrt{c} r / 2), & G(r) \text { in } \mathbb{C} P^{n}(c), \\
\sqrt{|c|} / 2, & H S, \\
(\sqrt{|c|} / 2) \tanh (\sqrt{|c|} r / 2), & G(r) \text { in } \mathbb{C} H^{n}(c), \\
(\sqrt{|c|} / 2) \operatorname{coth}(\sqrt{|c|} r / 2), & T(r) .\end{cases}\right.
$$

Here, we take a unit normal vector field $\mathcal{N}$ on $M$ in such a way that $\alpha$ is positive.

In this paper, we give characterisations of these totally $\eta$-umbilic real hypersurfaces. For a real hypersurface $M$ in $\widetilde{M}_{n}(c)$, we consider the following conditions on extrinsic shapes of geodesics:

(ES1) At each point $p$ of $M$, there exist orthonormal vectors $v_{1}, v_{2}, \ldots, v_{2 n-2} \in$ $T_{p} M$ orthogonal to $\xi_{p}$ satisfying the following:

(i) geodesics $\gamma_{i}=\gamma_{i}(s)(1 \leqq i \leqq 2 n-2)$ on $M$ with $\gamma_{i}(0)=p$ and $\dot{\gamma}_{i}(0)=v_{i}$ are mapped to circles of positive curvature in $\widetilde{M}_{n}(c)$,

(ii) geodesics $\gamma_{i j}=\gamma_{i j}(s)(1 \leqq i<j \leqq 2 n-2)$ on $M$ with $\gamma_{i j}(0)=p$ and $\dot{\gamma}_{i j}(0)=\left(v_{i}+v_{j}\right) / \sqrt{2}$ are mapped to circles of positive curvature in $\widetilde{M}_{n}(c)$.

(ES2) At each point $p \in M$, there exist orthonormal vectors $v_{1}, v_{2}, \ldots, v_{2 n-2} \in$ $T_{p} M$ orthogonal to $\xi_{p}$ satisfying that geodesics $\gamma_{i}=\gamma_{i}(s)(1 \leqq i \leqq 2 n-2)$ 
on $M$ with $\gamma_{i}(0)=p$ and $\dot{\gamma}_{i}(0)=v_{i}$ are mapped to circles of same positive curvature in $\widetilde{M}_{n}(c)$.

Weakening Condition (ES2), we also consider the following condition:

(ES2') At each point $p \in M$, there exist orthonormal vectors $v_{1}, v_{2}, \ldots, v_{2 n-2} \in$ $T_{p} M$ orthogonal to $\xi_{p}$, satisfying that geodesics $\gamma_{i}=\gamma_{i}(s)(1 \leqq i \leqq 2 n-2)$ on $M$ with $\gamma_{i}(0)=p$ and $\dot{\gamma}_{i}(0)=v_{i}$ are mapped to circles of positive curvature in $\widetilde{M}_{n}(c)$.

The difference between the conditions (ES2) and (ES2') is whether we suppose the curvatures of circles are the same or not. Our results are as follows.

THEOREM 1. Concerning a real hypersurface $M$ in $\mathbb{C} P^{n}(c)$ with $n \geqq 2$, the following five conditions are mutually equivalent.

(1) It is locally congruent to a geodesic hypersphere $G(r)(0<r<\pi / \sqrt{c})$.

(2) M satisfies condition (ES1).

(3) M satisfies condition (ES2), and has at most one congruence class of geodesics that are mapped to geodesics in $\mathbb{C} P^{n}(c)$ with respect to the isometry group $\mathrm{I}(M)$ of $M$.

(4) $M$ satisfies condition (ES2') and is positively curved.

(5) $M$ is non-negatively curved and the exterior derivative $d \eta$ of the contact form $\eta$ on $M$ satisfies $d \eta(X, Y)=k g(\phi X, Y)$ for all $X, Y \in T M$ with some non-zero constant $k$.

THEOREM 2. Concerning a real hypersurface $M$ in $\mathbb{C} H^{n}(c)$ with $n \geqq 2$, the following three conditions are mutually equivalent.

(1) $M$ is locally congruent to a totally $\eta$-umbilic real hypersurface.

(2) $M$ satisfies condition (ES1).

(3) $M$ satisfies condition (ES2).

THEOREM 3. A real hypersurface $M$ in $\mathbb{C} H^{n}(c)$ with $n \geqq 2$ is locally congruent to a geodesic hypersphere if and only if it satisfies the following two conditions:

(i) The exterior derivative $d \eta$ of the contact form $\eta$ on $M$ satisfies $d \eta(X, Y)=$ $k\langle\phi X, Y\rangle$ for all $X, Y \in T M$ with some non-zero constant $k$.

(ii) All sectional curvatures of $M$ satisfy $K>3 c / 4$.

3. Extrinsic shapes of geodesics. In this section, we study conditions on extrinsic shapes of geodesics on a real hypersurface in an $n$-dimensional non-flat complex space form $\widetilde{M}_{n}(c)$. For a smooth curve $\gamma$ on a real hypersurface $M$ in $\widetilde{M}_{n}(c)$, we call the curve $\iota \circ \gamma$ which is obtained through the isometric immersion $\iota: M \rightarrow \widetilde{M}_{n}(c)$ the extrinsic shape of $\gamma$. It is a basic idea to investigate a submanifold by observing how curves are mapped through its isometric immersion.

Generally, we consider a hypersurface $M$ of a Riemannian manifold $\widetilde{M}$. The Riemannian connections $\widetilde{\nabla}$ of $\widetilde{M}$ and $\nabla$ of $M$ are related by the following formulas of Gauss and Weingarten:

$$
\begin{aligned}
\widetilde{\nabla}_{X} Y & =\nabla_{X} Y+\langle A X, Y\rangle \mathcal{N}, \\
\widetilde{\nabla}_{X} \mathcal{N} & =-A X,
\end{aligned}
$$


for arbitrary vector fields $X$ and $Y$ on $M$, where $\langle$,$\rangle is the Riemannian metric of M$ induced from the metric on the ambient space $\widetilde{M}$ and $A$ is the shape operator of $M$ in $\widetilde{M}$. When $M$ is a real hypersurface of $\widetilde{M}=\widetilde{M}_{n}(c)$, as we have $\widetilde{\nabla} J=0$, these equalities show that

$$
\begin{aligned}
\left(\nabla_{X} \phi\right) Y & =\eta(Y) A X-\langle A X, Y\rangle \xi \\
\nabla_{X} \xi & =\phi A X .
\end{aligned}
$$

We call a smooth curve $\gamma$ parameterised by its arclength on a Riemannian manifold $M$ a circle if it satisfies $\nabla_{\dot{\gamma}} \dot{\gamma}=k Y, \nabla_{\dot{\gamma}} Y=-k \dot{\gamma}$ with some non-negative constant $k$ and a unit vector field $Y$ along $\gamma$. Clearly, a smooth curve $\gamma$ parameterised by its arclength is a circle if and only if it satisfies $\nabla_{\dot{\gamma}} \nabla_{\dot{\gamma}} \dot{\gamma}=-k^{2} \dot{\gamma}$ with some non-negative $k$.

Proposition 1. On a totally $\eta$-umbilic real hypersurface $M$ in $\widetilde{M}_{n}(c)$ having the shape operator $A=\alpha I+\beta \eta \otimes \xi$, every geodesic $\gamma=\gamma(s)$ on $M$ with initial vector $\dot{\gamma}(0)$ orthogonal to $\xi_{\gamma(0)}$ is mapped to a circle of the same positive curvature $\alpha$ in $\widetilde{M}_{n}(c)$.

Proof. As we have

$$
\frac{d}{d s}\left\langle\dot{\gamma}(s), \xi_{\gamma(s)}\right\rangle=\langle\dot{\gamma}(s), \phi A \dot{\gamma}(s)\rangle=\langle\dot{\gamma}(s), \phi(\alpha \dot{\gamma}(s)+\beta \eta(\dot{\gamma}(s)) \xi)\rangle=0
$$

by use of (3.4), we find that $\dot{\gamma}(s)$ is orthogonal to $\xi_{\gamma(s)}$ at every $s$. Therefore, we have $\widetilde{\nabla}_{\dot{\gamma}} \dot{\gamma}=\alpha \mathcal{N}$ and $\widetilde{\nabla}_{\dot{\gamma}} \mathcal{N}=-\alpha \dot{\gamma}$ by (3.1) and (3.2). We get the conclusion.

As can be seen in the above proof, for a geodesic $\gamma$ on a totally $\eta$-umbilic real hypersurface $M$ in $\widetilde{M}_{n}(c)$, the constant $\rho_{\gamma}=\langle\dot{\gamma}, \xi\rangle$ is an important invariant. We call this its structure torsion. Clearly, it satisfies $-1 \leqq \rho_{\gamma} \leqq 1$. Structure torsions of geodesics show their congruency. We say two geodesics are congruent to each other in strong sense with respect to the isometry group $\mathrm{I}(M)$ of $M$ if there exists $\varphi \in \mathrm{I}(M)$ satisfying $\gamma_{2}(s)=\left(\varphi \circ \gamma_{1}\right)(s)$ for all $s$. On a totally $\eta$-umbilic real hypersurface $M$ in $\widetilde{M}_{n}(c)$, two geodesics are congruent to each other in strong sense if and only if the absolute values of their structure torsions coincide (see Proposition 2.3 and Corollary 3.2 in [4]).

\section{PROPOSITION 2.}

(1) A geodesic hypersphere $G(r)$ of radius $r$ with $\pi /(2 \sqrt{c}) \leqq r<\pi / \sqrt{c}$ in $\mathbb{C} P^{n}(c)$ has just one congruence class of geodesics that are mapped to geodesics in the ambient space $\mathbb{C} P^{n}(c)$ with respect to the isometry group $\mathrm{I}(G(r))$ of $G(r)$.

(2) Other totally $\eta$-umbilic real hypersurfaces $M$ in $\widetilde{M}_{n}(c)$ has no geodesics that are mapped to geodesics in $\widetilde{M}_{n}(c)$.

Proof. We take a geodesic $\gamma$ on a totally $\eta$-umbilic real hypersurface $M$. We then have $\widetilde{\nabla}_{\dot{\gamma}} \dot{\gamma}=\left(\alpha+\beta \rho_{\gamma}^{2}\right) \mathcal{N}$. When $M=G(r)$ in $\mathbb{C} P^{n}(c)$, we find that the constant $\alpha+\beta \rho_{\gamma}^{2}$ is equal to $(\sqrt{c} / 2)\left\{\cot (\sqrt{c} r / 2)-\rho_{\gamma}^{2} \tan (\sqrt{c} r / 2)\right\}$. Since $\left|\rho_{\gamma}\right| \leqq 1$, we see that when $\pi /(2 \sqrt{c}) \leqq r<\pi / \sqrt{c}$, it is zero if and only if $\rho_{\gamma}= \pm \cot (\sqrt{c} r / 2)$, and that it is positive when $r<\pi /(2 \sqrt{c})$. When $M$ is a totally $\eta$-umbilic real hypersurface in $\mathbb{C} H^{n}(c)$, as both $\alpha$ and $\beta$ are positive, we see that $\alpha+\beta \rho_{\gamma}^{2}$ is always positive. We hence get the conclusion.

We here show a characterisation of totally $\eta$-umbilic real hypersurfaces in $\widetilde{M}_{n}(c)$ by extrinsic shapes of geodesics. For this sake, we here recall a characterisation of 
'standard real hypersurfaces' in a non-flat complex space form. In $\mathbb{C} P^{n}(c)(n \geqq 2)$, a Hopf hypersurface all of whose principal curvatures are constant is locally congruent to one of the following (see $[\mathbf{8}, \mathbf{1 7}])$ :

$\left(\mathrm{A}_{1}\right)$ a geodesic hypersphere of radius $r$, where $0<r<\pi / \sqrt{c}$,

$\left(\mathrm{A}_{2}\right)$ a tube $T_{\ell}(r)$ of radius $r$ around a totally geodesic $\mathbb{C} P^{\ell}(c)(1 \leqq \ell \leqq n-2)$, where $0<r<\pi / \sqrt{c}$,

(B) a tube $R(r)$ of radius $r$ around a complex hyperquadric $\mathbb{C} Q^{n-1}$, where $0<$ $r<\pi /(2 \sqrt{c})$

(C) a tube of radius $r$ around a $\mathbb{C} P^{1}(c) \times \mathbb{C} P^{(n-1) / 2}(c)$, where $0<r<\pi /(2 \sqrt{c})$ and $n(\geqq 5)$ is odd,

(D) a tube of radius $r$ around a complex Grassmannian $\mathbb{C G}_{2,5}$, where $0<r<$ $\pi /(2 \sqrt{c})$ and $n=9$,

(E) a tube of radius $r$ around a Hermitian symmetric space $S O(10) / U(5)$, where $0<r<\pi /(2 \sqrt{c})$ and $n=15$.

These real hypersurfaces are said to be of types $\left(A_{1}\right),\left(A_{2}\right),(B),(C),(D)$ and $(E)$. Unifying real hypersurfaces of types $\left(\mathrm{A}_{1}\right)$ and $\left(\mathrm{A}_{2}\right)$, we call them hypersurfaces of type (A). The numbers of distinct principal curvatures of these real hypersurfaces are 2, 3, $3,5,5$ and 5 , respectively.

In $\mathbb{C} H^{n}(c)(n \geqq 2)$, a Hopf hypersurface all of whose principal curvatures are constant is locally congruent to one of the following (see [5]);

$\left(\mathrm{A}_{0}\right)$ a horosphere in $\mathbb{C} H^{n}(c)$;

$\left(\mathrm{A}_{1,0}\right)$ a geodesic hypersphere of radius $r(0<r<\infty)$,

$\left(\mathrm{A}_{1,1}\right)$ a tube of radius $r$ around a totally geodesic $\mathbb{C} H^{n-1}(c)$, where $0<r<\infty$,

$\left(\mathrm{A}_{2}\right)$ a tube $T_{\ell}(r)$ of radius $r$ around a totally geodesic $\mathbb{C} H^{\ell}(c)(1 \leqq \ell \leqq n-2)$, where $0<r<\infty$,

(B) a tube $R(r)$ of radius $r$ around a totally real totally geodesic $\mathbb{R} H^{n}(c / 4)$, where $0<r<\infty$.

These real hypersurfaces are said to be of types $\left(A_{0}\right),\left(A_{1}\right),\left(A_{1}\right),\left(A_{2}\right)$ and $(B)$. We note that both geodesic hyperspheres and tubes around $\mathbb{C} H^{n-1}(c)$ are called of type $\left(\mathrm{A}_{1}\right)$. Unifying real hypersurfaces of types $\left(A_{0}\right),\left(A_{1}\right)$ and $\left(A_{2}\right)$, we call them hypersurfaces of type (A). The numbers of distinct principal curvatures of these real hypersurfaces are not greater than 3 . We shall call these real hypersurfaces as standard real hypersurfaces in a non-flat complex space form. For their principal curvatures, see $[\mathbf{5}, \mathbf{1 5}, \mathbf{1 8}]$. These standard real hypersurfaces are characterised in the following manner:

FACT $([3,6])$. A connected real hypersurface $M$ of $\widetilde{M}_{n}(c)$ is locally congruent to a standard real hypersurface if and only if it satisfies condition (ES2').

We should note that in the above characterisation, curvatures of extrinsic shapes of $\gamma_{i}(i=1, \ldots, 2 n-2)$ are not necessarily the same. We here study the relationship between principal curvatures and geodesics that are mapped to circles.

LEMMA 1. Let $M$ be a hypersurface isometrically immersed into a Riemannian manifold $\widetilde{M}$. A geodesic $\gamma$ on $M$ is mapped to a circle of positive curvature $k$ if and only if either $A \dot{\gamma}=k \dot{\gamma}$ or $A \dot{\gamma}=-k \dot{\gamma}$ holds.

Proof. By (3.1), we have $\widetilde{\nabla}_{\dot{\gamma}} \dot{\gamma}=\langle A \dot{\gamma}, \dot{\gamma}\rangle \mathcal{N}$. If $A \dot{\gamma}=k \dot{\gamma}$ or $A \dot{\gamma}=-k \dot{\gamma}$ holds with some positive $k$, we find by (3.2) that

$$
\widetilde{\nabla}_{\dot{\gamma}} \widetilde{\nabla}_{\dot{\gamma}} \dot{\gamma}= \pm k \widetilde{\nabla}_{\dot{\gamma}} \mathcal{N}=\mp k A \dot{\gamma}=-k^{2} \dot{\gamma}
$$


hence $\gamma$ is mapped to a circle of curvature $k$. On the other hand, if $\gamma$ is mapped to a circle of positive curvature $k$, then $\langle A \dot{\gamma}, \dot{\gamma}\rangle$ is constant. Thus, we have $\widetilde{\nabla}_{\dot{\gamma}} \widetilde{\nabla}_{\dot{\gamma}} \dot{\gamma}=\langle A \dot{\gamma}, \dot{\gamma}\rangle A \dot{\gamma}$. Hence, we obtain $\langle A \dot{\gamma}, \dot{\gamma}\rangle A \dot{\gamma}=-k^{2} \dot{\gamma}$. Since $k>0$, we obtain $A \dot{\gamma}=k \dot{\gamma}$ or $A \dot{\gamma}=$ $-k \dot{\gamma}$.

Proof of Theorem 1, 2 (Characterisations by extrinsic shapes of geodesics).

Propositions 1 and 2 show that (1) $\Rightarrow$ (2), (3) in Theorems 1 and 2.

(2) $\Rightarrow(1)$. By Lemma 1 , condition (ES1) shows that $v_{i}(1 \leqq i \leqq 2 n-2)$ and $v_{i}+$ $v_{j}(1 \leqq i<j \leqq 2 n-2)$ are principal curvature vectors. Therefore, they have the same principal curvature; hence, we find that all tangent vectors orthogonal to $\xi$ are principal. This guarantees that $\xi$ is principal and $M$ is $\eta$-umbilic in $\widetilde{M}_{n}(c)$.

(3) $\Rightarrow(1)$. By Fact and Lemma 1, the existence of geodesics that are mapped to circles shows that $M$ is a standard real hypersurface in $\widetilde{M}_{n}(c)$ and the principal curvatures $\lambda_{i}$ of $v_{i}(1 \leq i \leq 2 n-2)$ satisfy $\left|\lambda_{1}\right|=\cdots=\left|\lambda_{2 n-2}\right|$. Thus, if we restrict the shape operator $A$ to the sub-bundle $T^{0} M$ of tangent vectors orthogonal to $\xi$, then it has at most two distinct principal curvatures. We here give principal curvatures of hypersurfaces of types $\left(\mathrm{A}_{2}\right)$ and $(\mathrm{B})$ which correspond to vectors orthogonal to $\xi$ :

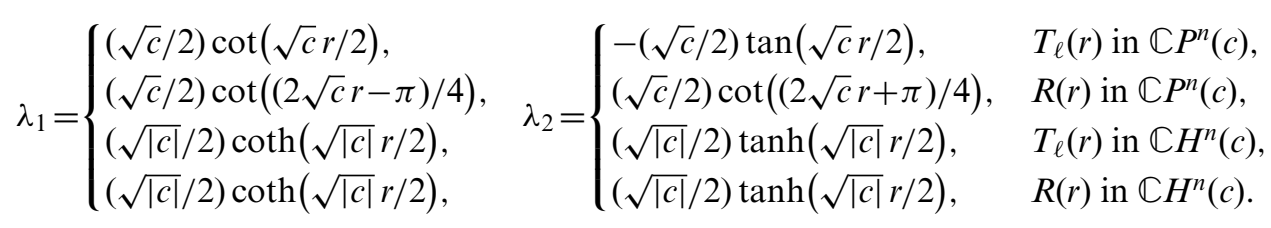

In view of principal curvatures given as above and those for totally $\eta$-umbilic real hypersurfaces given in the previous section, we find that in $\mathbb{C} H^{n}(c)$, the hypersurface $M$ is totally $\eta$-umbilic, and that in $\mathbb{C} P^{n}(c)$, it is either a geodesic hypersphere or a tube $T_{\ell}(\pi /(2 \sqrt{c}))$ of type $\left(\mathrm{A}_{2}\right)$.

We here study the tube $T_{\ell}(\pi /(2 \sqrt{c}))$. This tube has three distinct principal curvatures $\sqrt{c} / 2,-\sqrt{c} / 2,0$. Here, 0 is the principal curvature of $\xi$. For a geodesic $\gamma$ on $T_{\ell}(\pi /(2 \sqrt{c}))$, we decompose its velocity vector as $\dot{\gamma}(t)=X_{\gamma}(t)+Y_{\gamma}(t)+\rho_{\gamma} \xi_{\gamma(t)}$, where $A X_{\gamma}=(\sqrt{c} / 2) X_{\gamma}$ and $A Y_{\gamma}=-(\sqrt{c} / 2) Y_{\gamma}$. Since the shape operator $A$ of a hypersurface $M$ of type (A) satisfies

$$
\left\langle\left(\nabla_{X} A\right) Y, Z\right\rangle=-(c / 4)\{\eta(Y)\langle\phi X, Z\rangle+\eta(Z)\langle\phi X, Y\rangle\}
$$

for arbitrary vectors $X, Y, Z \in T M$ (see $[\mathbf{9}, \mathbf{1 5}]$ ), we find that $\frac{d}{d t}\langle A \dot{\gamma}, \dot{\gamma}\rangle=$ $\left\langle\left(\nabla_{\dot{\gamma}} A\right) \dot{\gamma}, \dot{\gamma}\right\rangle=0$. Therefore, we have $\langle A \dot{\gamma}, \dot{\gamma}\rangle=(\sqrt{c} / 2)\left(\left\|X_{\gamma}\right\|^{2}-\left\|Y_{\gamma}\right\|^{2}\right)$ is constant. As we have $\left\|X_{\gamma}\right\|^{2}+\left\|Y_{\gamma}\right\|^{2}=1-\rho_{\gamma}^{2}$, we find that both $\left\|X_{\gamma}\right\|$ and $\left\|Y_{\gamma}\right\|$ are constant along $\gamma$. We also find that the extrinsic shape of $\gamma$ is a geodesic if and only if $\left\|X_{\gamma}\right\|=\left\|Y_{\gamma}\right\|=\sqrt{\left(1-\rho_{\gamma}^{2}\right) / 2}$. It is clear that two geodesics are not congruent to each other if the absolute values of their structure torsions do not coincide. As a matter, of course, it is known that two geodesics $\gamma_{1}, \gamma_{2}$ on $T_{\ell}(r)$ are congruent to each other if their structure torsions satisfy $\left|\rho_{\gamma_{1}}\right|=\left|\rho_{\gamma_{2}}\right|$ and $\left\|X_{\gamma_{1}}\right\|=\left\|X_{\gamma_{2}}\right\|$ (see [1, 2]). Therefore, the tube $T_{\ell}(\pi /(2 \sqrt{c}))$ does not satisfy the condition that the number of congruence classes of geodesics that are mapped to circles is at most one. We hence conclude that a real hypersurface satisfying the conditions in (3) is totally $\eta$-umbilic.

Our proof also shows the following. 
THEOREM 4 ([13]). A real hypersurface $M$ in $\mathbb{C} P^{n}(c)$ is congruent to a tube $T_{\ell}(\pi /(2 \sqrt{c}))$ of type $\left(\mathrm{A}_{2}\right)$ if and only if it satisfies condition (ES2) and has more than one congruence class of geodesics that are mapped to geodesics in $\mathbb{C} P^{n}(c)$ with respect to the isometry group $\mathrm{I}(M)$ of $M$.

4. Sectional curvatures. We denote by $R$ the curvature tensor of a real hypersurface $M$ in $\widetilde{M}_{n}(c)$. The equation of Gauss is as follows:

$$
\begin{aligned}
\langle R(X, Y) Z, W\rangle= & \frac{c}{4}\{\langle Y, Z\rangle\langle X, W\rangle-\langle X, Z\rangle\langle Y, W\rangle+\langle\phi Y, Z\rangle\langle\phi X, W\rangle \\
& -\langle\phi X, Z\rangle\langle\phi Y, W\rangle-2\langle\phi X, Y\rangle\langle\phi Z, W\rangle\} \\
& +\langle A Y, Z\rangle\langle A X, W\rangle-\langle A X, Z\rangle\langle A Y, W\rangle .
\end{aligned}
$$

Therefore, the sectional curvature $K(X, Y)$ of the plane spanned by a pair $(X, Y)$ of orthonormal vectors is given as

$$
K(X, Y)=\frac{c}{4}\left(1+3\langle\phi X, Y\rangle^{2}\right)+\langle A X, X\rangle\langle A Y, Y\rangle-\langle A X, Y\rangle^{2} .
$$

To calculate sectional curvatures, we may suppose one of spanning vectors $X, Y$ does not have the component parallel to the characteristic vector $\xi$. We put $\rho_{X}=\langle X, \xi\rangle$ and $\rho_{Y}=\langle Y, \xi\rangle$. If they are not zero, we set

$$
\tilde{X}=\left(\rho_{X} X+\rho_{Y} Y\right) / \sqrt{\rho_{X}^{2}+\rho_{Y}^{2}}, \tilde{Y}=\left(\rho_{X} Y-\rho_{Y} X\right) / \sqrt{\rho_{X}^{2}+\rho_{Y}^{2}} .
$$

Then $\widetilde{X}, \widetilde{Y}$ are orthonormal, span the same plane as that spanned by $X, Y$, and $\rho_{\widetilde{Y}}=0$. Thus, we may suppose $\rho_{Y}=0$.

We now study sectional curvatures of a totally $\eta$-umbilic real hypersurface $M$ in $\widetilde{M}_{n}(c)$. Suppose its shape operator satisfy $A=\alpha I+\beta \eta \otimes \xi$. We take a pair of $(X, Y)$ of orthonormal tangent vectors that are orthogonal to $\xi$. We then find the sectional curvature of the plane spanned by $\sin \theta X+\cos \theta \xi$ and $Y$ is given as

$$
K(\sin \theta X+\cos \theta \xi, Y)=\frac{c}{4}\left\{1+3 \sin ^{2} \theta\langle\phi X, Y\rangle^{2}\right\}+\alpha^{2}+\alpha \beta \cos ^{2} \theta .
$$

If we write down individually by make use of values of $\alpha, \beta$ in Section 2 , we have the following (cf. [11]).

EXAMPLE 1 . When $M=G(r)$ in $\mathbb{C} P^{n}(c)$, we have

$$
K(\sin \theta X+\cos \theta \xi, Y)=\frac{c}{4}\left\{\sin ^{2} \theta\left(1+3\langle\phi X, Y\rangle^{2}\right)+\cot ^{2}(\sqrt{c} r / 2)\right\} .
$$

We hence find that sectional curvatures of $G(r)$ satisfy

$$
(c / 4) \cot ^{2}(\sqrt{c} r / 2) \leqq K \leqq c+(c / 4) \cot ^{2}(\sqrt{c} r / 2) .
$$

EXAMPLE 2. When $M=H S$ in $\mathbb{C} H^{n}(c)$, we have

$$
K(\sin \theta X+\cos \theta \xi, Y)=\frac{c}{4}\left\{\sin ^{2} \theta\left(1+3\langle\phi X, Y\rangle^{2}\right)-1\right\} .
$$

We find that sectional curvatures of $H S$ satisfy $3 c / 4 \leqq K \leqq-c / 4$. 
EXAMPLE 3. When $M=G(r)$ in $\mathbb{C} H^{n}(c)$, we have

$$
K(\sin \theta X+\cos \theta \xi, Y)=\frac{c}{4}\left\{\sin ^{2} \theta\left(1+3\langle\phi X, Y\rangle^{2}\right)-\operatorname{coth}^{2}(\sqrt{|c|} r / 2)\right\} .
$$

We find that sectional curvatures of $G(r)$ satisfy

$$
c-(c / 4) \operatorname{coth}^{2}(\sqrt{c} r / 2) \leqq K \leqq-(c / 4) \operatorname{coth}^{2}(\sqrt{c} r / 2) .
$$

EXAMPLE 4. When $M=T(r)$ in $\mathbb{C} H^{n}(c)$, we have

$$
K(\sin \theta X+\cos \theta \xi, Y)=\frac{c}{4}\left\{\sin ^{2} \theta\left(1+3\langle\phi X, Y\rangle^{2}\right)-\tanh ^{2}(\sqrt{|c|} r / 2)\right\} .
$$

We hence find that sectional curvatures of $T(r)$ satisfy

$$
c-(c / 4) \tanh ^{2}(\sqrt{c} r / 2) \leqq K \leqq-(c / 4) \tanh ^{2}(\sqrt{c} r / 2) .
$$

Here, we should note that in the above examples, each estimate on sectional curvatures not only gives an estimate but also shows the following: Given $K$ with $K_{\min } \leqq K \leqq K_{\max }$, there exists a plane $\alpha \subset T_{p} M$ at each point $p \in M$ satisfying $K(\alpha)=K$.

Proof of the third characterisation in Theorem 1. Proposition 1 and Examples 1-4 show that $(1) \Rightarrow(4)$ holds. We hence consider the converse. By condition (ES2), we find $M$ is a standard real hypersurface. We therefore study the curvature condition individually.

A real hypersurface $T_{\ell}(r)$ of type $\left(\mathrm{A}_{2}\right)$ has two distinct constant principal curvatures corresponding to vectors orthogonal to $\xi$. They are

$$
\lambda_{1}=(\sqrt{c} / 2) \cot (\sqrt{c} r / 2), \lambda_{2}=-(\sqrt{c} r / 2) \tan (\sqrt{c} r / 2)
$$

If we denote by $V_{\lambda_{i}}$ the sub-bundle of principal curvature vectors associated with $\lambda_{i}$, these sub-bundles $V_{\lambda_{1}}, V_{\lambda_{2}}$ are invariant under the action of $\phi$. We take orthonormal vectors $X \in V_{\lambda_{1}}$ and $Y \in V_{\lambda_{2}}$. We then find that $K(X, Y)=0$ by (4.2).

A real hypersurface $R(r)$ of type (B) has also two distinct constant principal curvatures corresponding to vectors orthogonal to $\xi$, which are

$$
\lambda_{1}=(\sqrt{c} / 2) \cot (\sqrt{c} r / 2-\pi / 4), \lambda_{2}=(\sqrt{c} / 2) \cot (\sqrt{c} r / 2+\pi / 4) .
$$

The sub-bundles $V_{\lambda_{1}}, V_{\lambda_{2}}$ of principal curvature vectors associated with $\lambda_{1}, \lambda_{2}$ satisfy $\phi\left(V_{\lambda_{1}}\right)=V_{\lambda_{2}}, \phi\left(V_{\lambda_{2}}\right)=V_{\lambda_{1}}$. Taking a unit vector $Y \in V_{\lambda_{1}}$, we find that

$$
K(\xi, Y)=-\frac{c(2+\sin \sqrt{c} r)}{4 \sin \sqrt{c} r}<0 .
$$

Real hypersurfaces of types (C), (D) and (E) have four distinct constant principal curvatures corresponding to vectors orthogonal to $\xi$. They are

$$
\begin{array}{ll}
\lambda_{1}=(\sqrt{c} / 2) \cot (\sqrt{c} r / 2-\pi / 4), & \lambda_{2}=(\sqrt{c} / 2) \cot (\sqrt{c} r / 2+\pi / 4), \\
\lambda_{3}=(\sqrt{c} / 2) \cot (\sqrt{c} r / 2), & \lambda_{4}=-(\sqrt{c} / 2) \tan (\sqrt{c} r / 2),
\end{array}
$$


if their radii as tubes are $r$. Sub-bundles associated with these principal curvatures satisfy $\phi\left(V_{\lambda_{1}}\right)=V_{\lambda_{2}}, \phi\left(V_{\lambda_{2}}\right)=V_{\lambda_{1}}, \phi\left(V_{\lambda_{3}}\right)=V_{\lambda_{3}}$ and $\phi\left(V_{\lambda_{4}}\right)=V_{\lambda_{4}}$. If we take a unit vector $Y \in V_{\lambda_{1}}$, we obtain the expression (4.3) on the sectional curvature of a plane spanned by $\xi$ and $Y$. Thus, we find that real hypersurfaces of types $\left(\mathrm{A}_{2}\right),(\mathrm{B}),(\mathrm{C}),(\mathrm{D})$ and $(\mathrm{E})$ are not positively curved. We hence get the conclusion.

5. Exterior derivative of the contact form. In this section, we characterise totally $\eta$-umbilic real hypersurfaces in a non-flat complex space form by using a property of exterior derivatives of their contact forms.

For the contact form $\eta$ on a real hypersurface $M$ of $\tilde{M}_{n}(c)$, we consider its exterior derivative $d \eta$, which is given by

$$
d \eta(X, Y):=(1 / 2)\{X(\eta(Y))-Y(\eta(X))-\eta([X, Y])\} .
$$

The equality (3.4) implies that

$$
d \eta(X, Y)=\frac{1}{2}\langle(\phi A+A \phi) X, Y\rangle .
$$

This equation means that there exist no real hypersurfaces having the closed contact form $\eta$ in $\widetilde{M}_{n}(c)$ (see Corollary 2.12 in [15]). For totally $\eta$-umbilic real hypersurfaces, (5.1) shows the following.

Proposition 3. Let $M$ be a totally $\eta$-umbilic real hypersurface of $\tilde{M}_{n}(c)$ having the shape operator $A=\alpha I+\beta \eta \otimes \xi$. The exterior derivative $d \eta$ of its contact form $\eta$ satisfies $d \eta(X, Y)=\alpha\langle\phi X, Y\rangle$ for all vectors $X, Y \in T M$.

We are interested in the converse of this result.

Proposition 4. A real hypersurface $M$ in $\widetilde{M}_{n}(c)$ is either totally $\eta$-umbilic or of type (B) if and only if there is a non-zero constant $k$ satisfying $d \eta(X, Y)=k\langle\phi X, Y\rangle$ for all $X, Y \in T M$.

Proof. We first consider the 'only if' part. We only need to investigate this for real hypersurfaces of type (B). For a real hypersurface $M$ of type (B), the subbundle $T^{0} M=\{X \in T M \mid\langle X, \xi\rangle=0\}$ is decomposed into two sub-bundles $V_{\lambda_{1}}, V_{\lambda_{2}}$ of principal curvature vectors associated with principal curvatures $\lambda_{1}, \lambda_{2}$, and these satisfy $\phi\left(V_{\lambda_{1}}\right)=V_{\lambda_{2}}, \phi\left(V_{\lambda_{2}}\right)=V_{\lambda_{1}}$. Therefore, we see $\phi A+A \phi=\left(\lambda_{1}+\lambda_{2}\right) \phi$. Thus, we obtain $d \eta(X, Y)=\frac{1}{2}\left(\lambda_{1}+\lambda_{2}\right)\langle\phi X, Y\rangle$ for all $X, Y \in T M$.

We next study the 'if' part. We shall determine a real hypersurface $M$ whose shape operator $A$ satisfies $\phi A+A \phi=2 k \phi$. Clearly, we have $\phi A \xi=0$, which shows that $\xi$ is principal. For a Hopf hypersurface, it is known that the principal curvature $\delta$ of $\xi$ is locally constant on $M$ and every principal curvature vector $X$ orthogonal to $\xi$ satisfies $(2 \lambda-\delta) A \phi X=(\delta \lambda+(c / 2)) \phi X$, where $\lambda$ denotes the principal curvature of $X$ (see $[9,15])$. Making use of the relation $\phi A+A \phi=2 k \phi$, we see $X$ satisfies $(2 \lambda-\delta)(2 k-\lambda) \phi X=(\delta \lambda+(c / 2)) \phi X$. As $\phi X \neq 0$, we find that $\lambda$ satisfies the following quadratic equation:

$$
4 \lambda^{2}-8 k \lambda+c+4 k \delta=0
$$

Since $k$ and $\delta$ are constant, this shows that $\lambda$ is also constant on the connected real hypersurface $M$. Thus, we can see that our real hypersurface is a Hopf hypersurface 
with at most three distinct constant principal curvatures. So, we find that $M$ is of type either (A) or (B). If we suppose $M$ is of type $\left(\mathrm{A}_{2}\right)$, then $T^{0} M$ is decomposed into two sub-bundles $V_{\lambda_{1}}, V_{\lambda_{2}}$ of principal curvature vectors that are invariant under the action of $\phi$. Taking non-zero vectors $X \in V_{\lambda_{1}}, \quad Y \in V_{\lambda_{2}}$, we see $(\phi A+A \phi)(X+Y)=$ $2\left(\lambda_{1} \phi X+\lambda_{2} \phi Y\right)$. Therefore, real hypersurfaces of type $\left(\mathrm{A}_{2}\right)$ do not satisfy the condition $\phi A+A \phi=2 k \phi$. We hence get the conclusion.

Proof of Theorem 1 (Exterior derivatives of contact forms). Proposition 3 and Example 3 show that every geodesic hypersphere $M$ is positively curved and the exterior derivative of the contact form satisfies $d \eta(X, Y)=\alpha\langle\phi X, Y\rangle$ for all vectors $X, Y \in T M$. On the other hand, by Proposition 4, we see that $M$ is either totally $\eta$-umbilic or of type (B). As can be seen in the previous section, real hypersurfaces of type (B) are not non-negatively curved. This completes the proof of Theorem 1.

Proof of Theorem 3. When we take a geodesic hypersphere, Example 3 shows that sectional curvatures satisfy $K>3 c / 4$ and Proposition 3 shows that the exterior derivative of the contact form satisfies $d \eta(X, Y)=\alpha\langle\phi X, Y\rangle$ for all vectors $X, Y \in$ $T M$. On the other hand, by Proposition 4, we see that $M$ is either totally $\eta$-umbilic or of type (B). Examples 2 and 4 show that horospheres and tubes around $\mathbb{C} H^{n-1}$ do not satisfy the curvature condition. We hence check sectional curvatures of real hypersurfaces of type (B) in $\mathbb{C} H^{n}(c)$. A real hypersurface $R(r)$ of type (B) has two distinct principal curvatures corresponding to vectors orthogonal to $\xi$, which are

$$
\lambda_{1}=(\sqrt{|c|} / 2) \operatorname{coth}(\sqrt{|c|} r / 2), \lambda_{2}=(\sqrt{|c|} / 2) \tanh (\sqrt{|c|} r / 2)
$$

If we take a unit vector $X$ satisfying $A X=\lambda_{1} X$, we have $A \phi X=\lambda_{2} \phi X$. Hence, we find by (4.2) that $K(X, \phi X)=3 c / 4$. We therefore get the conclusion.

As an immediate consequence of Theorem 3, we obtain the following:

Proposition 5. A real hypersurface $M$ of $\mathbb{C} H^{n}(c), n \geqq 2$ is locally congruent to a geodesic hypersphere $G(r)$ of radius $r(0<r \leqq \log 3 / \sqrt{|c|})$ in $\mathbb{C} H^{n}(c)$ if and only if $M$ is non-negatively curved and the exterior derivative $d \eta$ of the contact form $\eta$ on $M$ satisfies $d \eta(X, Y)=k g(\phi X, Y)$ for all $X, Y \in T M$ with some non-zero constant $k$.

Proof. Solving the following inequality

$$
c-(c / 4) \operatorname{coth}^{2}(\sqrt{|c|} r / 2) \geqq 0,
$$

we obtain the conclusion (see Theorem 3 and Example 3).

ACKNOWLEDGEMENTS. The first author is partially supported by Grant-in-Aid for Scientific Research (C) (No. 23540097), Japan Society for the Promotion of Sciences. The second author is partially supported by Grant-in-Aid for Scientific Research (C) (Nos. $20540071 \&$ 24540075), Japan Society for the Promotion of Sciences. The third author is partially supported by Basic Science Research Program through the National Research Foundation of Korea (NRF) funded by the Ministry of Education, Science and Technology (2010-0007184). 


\section{REFERENCES}

1. T. Adachi, Geodesics on real hypersurfaces of type $A_{2}$ in a complex space form, Mon. Math. 153 (2008), 283-293.

2. T. Adachi and S. Maeda, A congruence theorem of geodesics on some naturally reductive Riemannian homogeneous manifolds, C. R. Math. Rep. Acad. Sci. Canada 26 (2004), 11-17.

3. T. Adachi, S. Maeda and M. Kimura, A characterization of all homogeneous real hypersurfaces in a complex projective space by observing the extrinsic shape of geodesics, Archiv der Math. 73 (1999), 303-310.

4. T. Adachi, S. Maeda and M. Yamagishi, Length spectrum of geodesic spheres in a non-flat complex space form, J. Math. Soc. Japan 54 (2002), 373-408.

5. J. Berndt, Real hypersurfaces with constant principal curvatures in complex hyperbolic space, J. Reine Angew. Math. 395 (1989), 132-141.

6. B. Y. Chen and S. Maeda, Hopf hypersurfaces with constant principal curvatures in complex projective or complex hyperbolic spaces, Tokyo J. Math. 24 (2001), 133-152.

7. D. Ferus and S. Schirrmacher, Submanifolds in Euclidean space with simple geodesics, Math. Ann. 260 (1982), 57-62.

8. M. Kimura, Real hypersurfaces and complex submanifolds in complex projective space, Trans. Amer. Math. Soc. 296 (1986), 137-149.

9. Y. Maeda, On real hypersurfaces of a complex projective space, J. Math. Soc. Japan 28 (1976), 529-540.

10. S. Maeda, Real hypersurfaces of complex projective spaces, Math. Ann. 263 (1983), 473-478.

11. S. Maeda and M. Kimura, Sectional curvatures of some homogeneous real hypersurfaces in a complex projective space, Complex Analysis and Mathematical Physics, in Proceedings of the 8th international workshop on complex structures and vector fields, (World Scientific, Bulgaria, 2007).

12. S. Maeda and K. Ogiue, Characterizations of geodesic hyperspheres in a complex projective space by observing the extrinsic shape of geodesics, Math. Z. 225 (1997), 537-542.

13. S. Maeda and K. Okumura, Three real hypersurfaces some of whose geodesics are mapped to circles with the same curvature in a nonflat complex space form, Geom. Dedicata 156 (2012), 71-80.

14. S. Montiel, Real hypersurfaces of a complex hyperbolic space, J. Math. Soc. Japan 37 (1985), 515-535.

15. R. Niebergall and P. J. Ryan, Real hypersurfaces in complex space forms, in Tight and taut submanifolds (Cecil T. E. and Chern S. S., Editors) (Cambridge University Press, New York, 1997), 233-305.

16. K. Sakamoto, Planar geodesic immersions, Tôhoku Math. J. 29 (1977), 25-56.

17. R. Takagi, On homogeneous real hypersurfaces in a complex projective space, Osaka J. Math. 10 (1973), 495-506.

18. R. Takagi, Real hypersurfaces in a complex projective space with constant principal curvatures, J. Math. Soc. Japan 27 (1975), 43-53. 